\title{
A revised stent-retriever anchor technique to obtain the distal access through a large or giant aneurysm
}

\author{
Emilio Lozupone ${ }^{1}$, Giuseppe Garignano ${ }^{2}$, Francesco D’Argento ${ }^{2}$, Andrea Alexandre², lacopo Valente ${ }^{2}$, \\ Pietro Trombatore ${ }^{3}$, Alessandro Pedicelli ${ }^{2}$ \\ 'UOC Neuroradiologia Diagnostica ed Interventistica, Azienda Ospedaliera Vito Fazzi, Lecce 73100, Italy. \\ ${ }^{2}$ Dipartimento di Diagnostica per Immagini, Radioterapia Oncologica ed Ematologia, UOC Radiologia e Neuroradiologia, \\ Fondazione Policlinico Universitario A.Gemelli IRCCS, Rome 00168, Italy. \\ ${ }^{3}$ Università Cattolica del Sacro Cuore, Rome 00168, Italy.
}

Correspondence to: Dr. Emilio Lozupone, UOC Neuroradiologia Diagnostica ed Interventistica, Azienda Ospedaliera Vito Fazzi, Piazza Filippo Muratore 1, Lecce 73100, Italy. E-mail: emilio.lozupone@live.it

How to cite this article: Lozupone E, Garignano G, D'Argento F, Alexandre A, Valente I, Trombatore P, Pedicelli A. A revised stent-retriever anchor technique to obtain the distal access through a large or giant aneurysm. Vessel Plus 2021;5:24.

https://dx.doi.org/10.20517/2574-1209.2020.97

Received: 22 Dec 2020 First Decision: 22 Jan 2021 Revised: 30 Jan 2021 Accepted: 18 Feb 2021 Published: 13 May 2021

Academic Editor: Elisa F. M. Ciceri Copy Editor: Yue-Yue Zhang Production Editor: Xi-Jun Chen

\begin{abstract}
Endovascular treatment of brain aneurysms with flow diverter device can be technically difficult. Obtaining distal access through the wide neck of a large or giant aneurysm may be challenging and sometimes the microcatheter needs to be looped inside the aneurysm. However, the inability to resolve this loop and to straighten the microcatheter can preclude flow diverter placement. This brief report presents four cases of large/giant aneurysms treated with flow diverter device, in which our innovative stent-retriever anchor technique was used to obtain the distal access of the parent artery. All procedures were technically successful, and no complications were recorded.
\end{abstract}

Keywords: Flow diverter device, anchor technique, stent-retriever, large or giant cerebral aneurysm, endovascular treatment

\section{INTRODUCTION}

Endovascular treatment of large or giant brain aneurysms remains a challenge for endovascular strategies ${ }^{[1,2]}$. 
The flow diverter device (FDD) has radically changed the natural history of these types of aneurysm in terms of rate of recanalization, morbidity, and mortality ${ }^{[3]}$. To deploy a flow diverter device, a stable distal access across the aneurysm neck must be achieved.

However, reaching the distal artery arising from the aneurysm could be technically difficult due to the distorted anatomy of the aneurysm, the acute angulation between the inflow and outflow vessels, or the presence of stenosis of the distal parent artery ${ }^{[3]}$. In these situations, the microcatheter needs to be looped inside the aneurysm in order to overcome the aneurysm's neck. Resolving the loop of the microcatheter within the aneurysm is not always easy, and the inability to straighten the microcatheter can preclude the correct deployment of $\mathrm{FDD}^{[4]}$.

Few techniques have previously been described to solve this problem as the "balloon", "stent", "Solitaire", and "sea anchor" techniques ${ }^{[4-7]}$.

We report a small retrospective series in which a low-profile stent-retriever (Aperio ${ }^{\circledR}$, Acandis $\mathrm{GmbH}$, Pforzheim, Germany), deliverable with a lightweight microcatheter, allowed a successful reduction of a looped microcatheter within an aneurysm.

\section{CASE REPORT}

\section{Patients}

Between January 2016 and December 2019, 335 brain aneurysms (ruptured and unruptured) were treated at our institution. Written informed consent for the procedure was obtained from all patients before they underwent the endovascular treatment.

In this retrospective study, the authors reviewed clinical, demographic, and procedural data of four patients ( 3 females and 1 male; age range 51-73) with a large or giant brain aneurysm treated with FDD, in which the described stent-retriever anchor technique was used. Intra-procedural complications were recorded, highlighting technique-related complications, namely vasospasm/injuries in the distal parent vessel or aneurysm bleeding.

\section{Stent-retriever anchor technique}

After gaining access to the femoral artery, selective catheterization of the parent vessel was performed using a long sheath introducer and an intermediate catheter. Then, a pre-curved lightweight microcatheter (0.0165" coil microcatheter) was looped inside the aneurysmal sac in order to overcome the neck of the aneurysm [Figure 1A]. Once the microcatheter tip had overcome the aneurysm and reached the outflow vessel, an Aperio stent-retriever (3.5 mm × $28 \mathrm{~mm}$; Acandis GmbH, Pforzheim Germany) was delivered through the microcatheter. The Aperio ${ }^{\circledR}$ stent-retriever was then fully deployed in the distal landing zone of the vessel [Figure $1 \mathrm{~B}$ ] and the microcatheter was gently retracted in order to resolve the loop [Figure $1 \mathrm{C}$ ]. Consequently, the 0.0165 " microcatheter was removed [Figure 1D] and the 0.027 " microcatheter was delivered into the distal vessel over the pushing wire of the Aperio ${ }^{\circ}$ stent-retriever [Figure 1E]. Then, the Aperio $^{\circ}$ stent-retriever was re-sheathed and the $0.027^{\prime \prime}$ microcatheter was used to deploy the flow diverter device [Figure $1 \mathrm{~F}$ ].

\section{Results}

Three aneurysms were unruptured and one was previously ruptured [Table 1]. Two patients were asymptomatic (the aneurysm was an incidental discovery), one patient presented with a complete deficit of III c.n. [Figure 2], and one patient presented with SAH. The latter (Patient 4) was a giant aneurysm of the 
Table 1. Clinical, demographic, and procedural data

\begin{tabular}{|c|c|c|c|c|c|c|c|c|c|c|c|}
\hline \multirow{2}{*}{ Patients } & \multirow{2}{*}{ Sex } & \multirow{2}{*}{ Age } & \multirow{2}{*}{ Symptomology } & \multicolumn{3}{|c|}{ Aneurysm } & \multicolumn{3}{|c|}{ Procedure } & \multicolumn{2}{|c|}{$\begin{array}{l}\text { Intraprocedural } \\
\text { complication }\end{array}$} \\
\hline & & & & Location & $\begin{array}{l}\text { Size } \\
(\mathrm{mm})\end{array}$ & $\begin{array}{l}\text { Dome/neck } \\
\text { ratio }\end{array}$ & & FDD & Coils & $\begin{array}{l}\text { Aneurysm } \\
\text { rupture }\end{array}$ & Vasospasm \\
\hline 1 & $M$ & 73 & None & Paraophthalmic & 16 & 1.3 & Triaxial & FRED & Yes & No & No \\
\hline 2 & $\mathrm{~F}$ & 51 & None & Paraophthalmic & 15 & 1.7 & Triaxial & I PED & Yes & No & No \\
\hline 3 & $\mathrm{~F}$ & 51 & Left III c.n. deficit & Basilar Tip & 34 & 2.1 & Triaxial & I DERIVO & No & No & No \\
\hline 4 & $\mathrm{~F}$ & 52 & Previous SAH & Paraophthalmic & 26 & 2.6 & Triaxial & | FRED & Yes & No & No \\
\hline
\end{tabular}
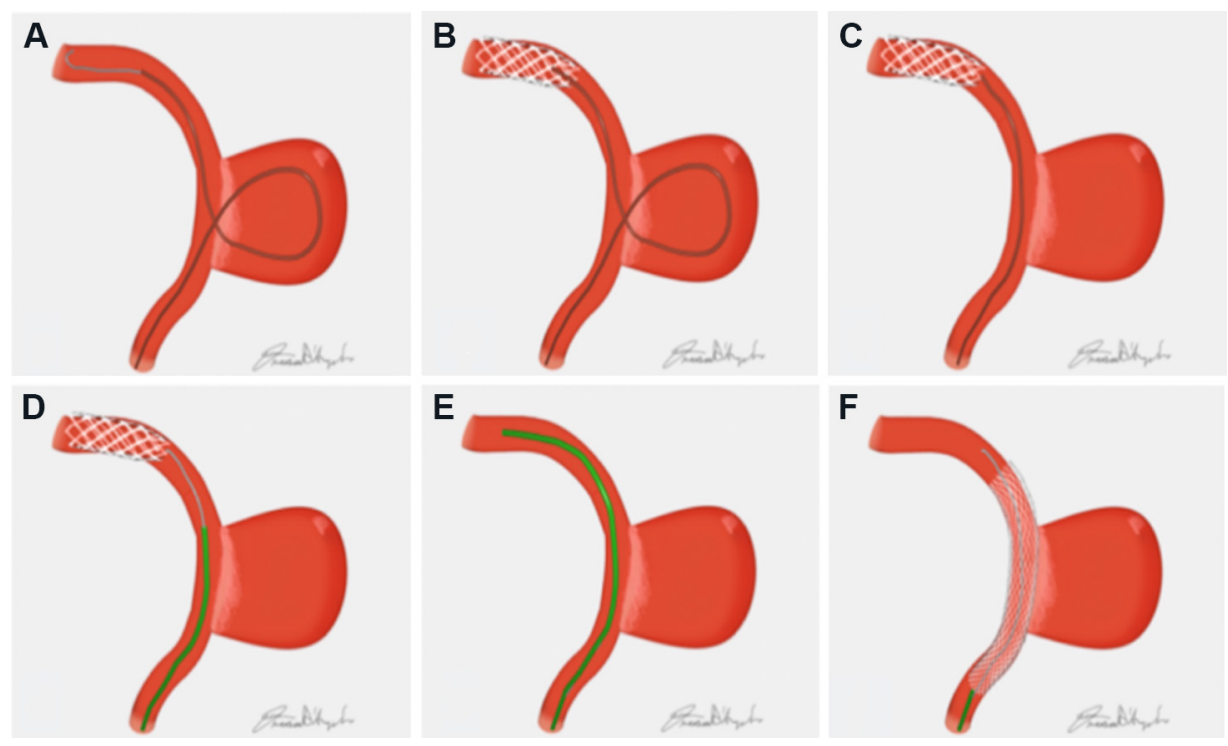

Figure 1. Diagram depicting the Aperio ${ }^{\circledR}$ stent-retriever anchor technique for navigating a large/giant aneurysm: (A) $0.0165^{\prime \prime}$ microcatheter looped inside the aneurysm; (B) deployment of the Aperio ${ }^{\circledR}$ stent-retriever in the outflow vessel through the $0.0165^{\prime \prime}$ microcatheter; (C) resolving the $0.0165^{\prime \prime}$ microcatheter loop; (D) delivering of the $0.027^{\prime \prime}$ microcatheter over the pushing wire of the Aperio ${ }^{\circledR}$ stent-retriever; (E) re-sheathing and removing of the Aperio ${ }^{\circledR}$ stent-retriever; and (F) deployment of flow diverter device.

paraophthalmic segment of the left ICA that was first partially embolized with platinum coils; after the complete recover of the patient ( 3 months later), the treatment was completed by deploying a FDD. Three aneurysms belonged to the anterior circulation (paraophthalmic segment) and one to the posterior circulation (basilar tip). The average size of the aneurysms was $23 \mathrm{~mm}$ (range 15-34 $\mathrm{mm}$ ) with a mean dome height/neck ratio of 1.9 (range 1.6-2.6).

Dual antiplatelet therapy was started seven days before the procedure (ASA $100 \mathrm{mg}$ and clopidogrel $75 \mathrm{mg}$ ) and was continued for 12 months. In all cases, a triaxial system was used to perform the procedure. In three cases, platinum coils were released in the aneurysm, using the "jailing" technique. No intra-procedural and post-procedural complications were recorded.

\section{DISCUSSION}

The treatment of large and giant wide-neck aneurysms remains a challenge for endovascular strategies ${ }^{[1]}$. FDD has radically changed the endovascular management of these aneurysms in terms of recanalization rate, morbidity, and mortality ${ }^{[2,3]}$. FDDs are a new generation stents characterized by a low porosity that allows them to achieve aneurysmal occlusion by reduction of the blood flow within the aneurysm and by the 

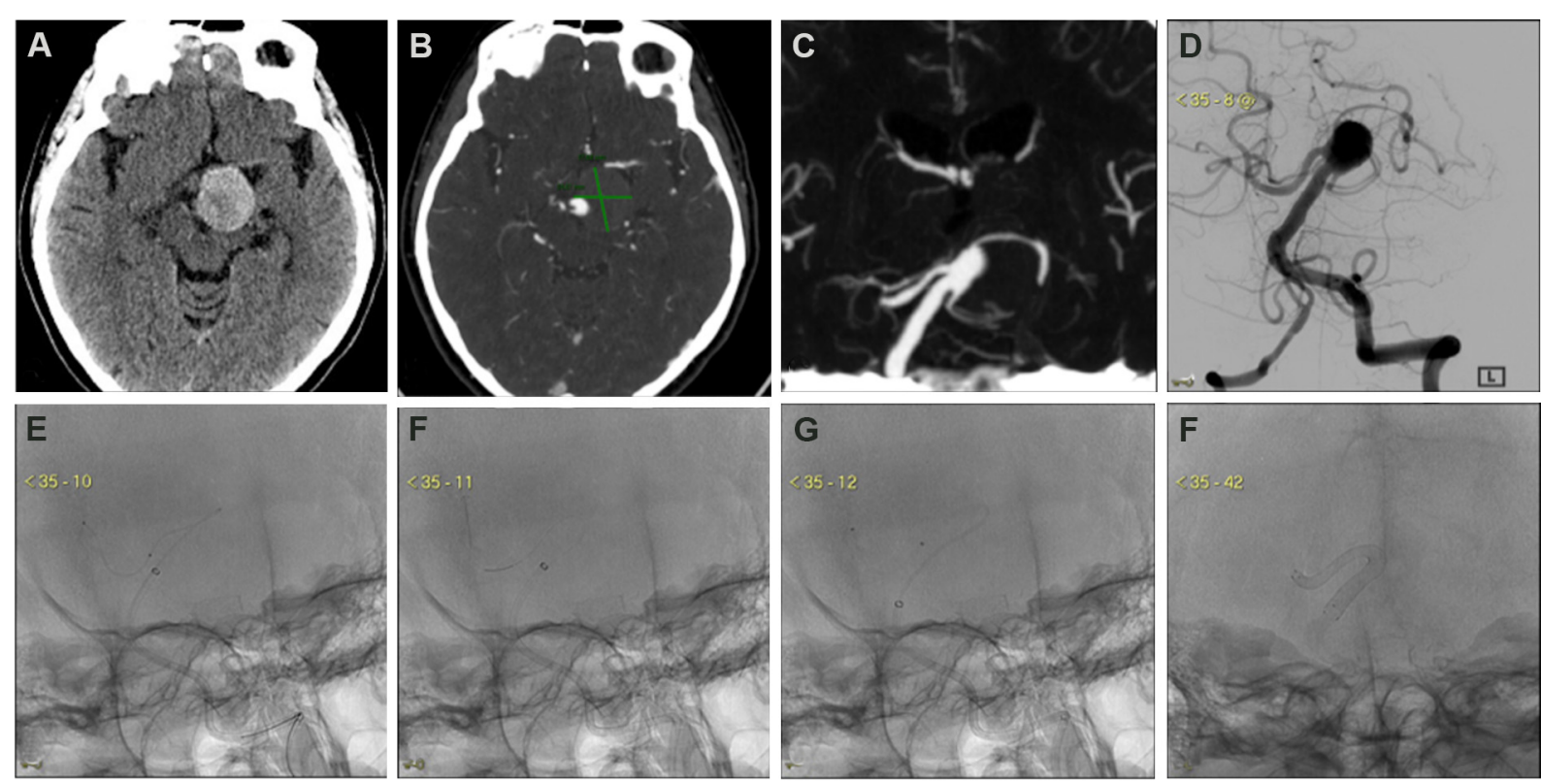

Figure 2. A 52-year-old woman with a rapid onset of deficit of left III cranial nerve. Non-enhanced CT (A) and CT-angio images (B, C) showed a partially thrombosed giant aneurysm of the basilar tip. A frontal angiogram (D) better defined the anatomy of the aneurysm with an acute angulation between the basilar artery and the right PCA [distal landing zone for the flow diverter device (FDD)]. A 0.0165" microcatheter was navigated and looped inside the aneurysm to gain the right PCA (E). An Aperio stent retriever ( $3.5 \mathrm{~mm}$ ' $28 \mathrm{~mm}$ ) was fully deployed in the PCA and the microcatheter was removed (F). Over the pushing wire of the stent-retriever, the 0.027" microcatheter was delivered in the right PCA $(G)$. The subtracted frontal image $(H)$ showed the correct deployment of the FDD at the end of the procedure.

promotion of reendothelialization across the neck of the aneurysm.

Nevertheless, the reconstruction of the parent artery with the FDD can be technically difficult. Reaching the distal parent vessels of the aneurysm can be complicated by the unfavorable anatomy of the aneurysm, the acute angulation between the inflow and outflow vessels, or the presence of stenosis the distal parent artery. In such situations, the microcatheter needs to be looped inside the sac in order to overcome the aneurysm neck. This maneuver is particularly difficult to perform with a $0.027 \% / 0.021$ " microcatheter, necessary for the deployment of FFDs, which are clearly stiffer than a $0.017 \% / 0.0165 "$ microcatheter ${ }^{[8]}$.

Resolving the loop is thus mandatory to deploy the FDD. Attempting to straighten the loop by withdrawing the catheter will frequently result in prolapse of the microcatheter tip out of the distal vessel and into the aneurysm dome, due to a lack of adequate support within the distal vasculature ${ }^{[6]}$. Different anchor techniques can be used to straighten the microcatheter. Many techniques have previously been described to address this problem, such as the "balloon", "stent", "Solitaire", and "sea anchor" techniques ${ }^{[4-7]}$.

"Balloon technique" relies on the use of a balloon microcatheter, which is inflated distally to the aneurysm, in order to straighten the microcatheter; injuries of the distal artery access and thromboembolic complications could potentially occur during balloon inflation ${ }^{[4]}$.

Edwards et al..$^{[6]}$ described one case of a large basilar tip aneurysm in which a detachable coil was advanced in the cerebral posterior artery and then used as an anchor for straightening of the looped microcatheter. 
Fargen et al. ${ }^{[5]}$ resolved the looped microcatheter using a partial retrievable stent as an anchor in the distal access.

Parry et al. ${ }^{[7]}$ described the Solitaire (Medtronic) salvage technique, which allowed the successful reduction of the looped catheter within the aneurysm in three cases. In all cases, once the reduction maneuver was completed, the Solitaire was re-sheathed and the microcatheter was exchanged using an exchange microwire $^{[7]}$.

Based on the principle of the Solitaire technique, we present our salvage technique that relies on a new generation of low-profile stent-retriever (Aperio ${ }^{\circ}$, Acandis $\mathrm{GmbH}$, Pforzheim, Germany), which can be delivered through a lightweight microcatheter (inner diameter 0.0165"/0.017").

Quäschling et al. ${ }^{[8]}$ recently described the safety and feasibility of this technique with another low-profile stent-retriever (pREset LITE, Phenox, Bochum, Germany) in a case series of 14 patients.

However, we assume that the greater length of the Aperio ${ }^{\circledR}$ stent-retriever compared to the pREset LITE ${ }^{\circledR}$ stent-retriever ( $30 \mathrm{~mm} v s .20 \mathrm{~mm}$ ) could theoretically guarantee a more stable distal anchorage during the several maneuvers. The anchor technique presents two additional advantages compared to the Solitaire salvage technique used by Parry et al. ${ }^{[7]}$. Firstly, the use of a smaller microcatheter allows an easier and safer intra-aneurismal navigation, reducing stress force on the aneurysm walls. Furthermore, with this technique, the 0.027 " microcatheter is delivered over the pushing wire of the stent-retriever and an exchange wire is not required. The pushing wire of the Aperio ${ }^{\circ}$ stent-retriever is insufficient for a traditional exchange maneuver $(212 \mathrm{~cm})$. However, the exchange of the microcatheter is possible by taking advantage of the stent radial force that anchors the stent itself into the vessel, avoiding its displacement during the removal of the 0.0165 " microcatheter and the delivery of the 0.027 " microcatheter in the outflow vessel over the pushing wire.

As previously reported, there may be concerns about placing a stent-retriever in a healthy artery which can cause dissection or vasospasm ${ }^{[7]}$. To prevent this possible complication, our suggestion is to position the stent in the M1 segment for the anterior circulation and the P2 segment for the posterior circulation, avoiding the deployment of the stent into more distal and smaller branches that are definitely more fragile. However, in our series, we did not record any artery injuries during the procedure or at the angiographic follow-up.

Treatment of large and giant brain aneurysms with flow diverter devices can be technically challenging, especially when distal access can be obtained only through aneurysm navigation ${ }^{[1]}$. In these situations, when standard maneuvers fail, a stent-retriever can be used as an anchor to permit straightening of the looped microcatheter and delivery of the 0.027 " microcatheter over the pusher wire of the stent-retriever.

\section{Conclusion}

Although endovascular treatment of large and giant aneurysms with flow diverter can be technically difficult, the presented technique represents a useful and safe tool to maintain the distal access when looping of the microcatheter inside the aneurysm is necessary.

\section{DECLARATIONS}

\section{Authors' contributions}

Contribute in conception and design of the study: Lozupone E, Pedicelli A 
Contribute in writing and reviewing of the manuscript: Garignano $G$

Contribute to data acquisition: D'Argento F, Alexandre A, Valente I, Trombatore P

\section{Availability of data and materials}

The data of this paper are available on request at the corresponding author.

\section{Financial support and sponsorship}

None.

\section{Conflicts of interest}

All authors declared that there are no conflict of interest.

\section{Ethical approval and consent to participate}

Institutional review board approval was obtained for this retrospective study. Protocol ID 3583, approved on 5 November 2020 by ethical committee of fondazione policlinico universitario A.Gemelli IRCCS, Rome, Italy. All procedures performed were in accordance with the ethical standards of the institutional and/or national research committee and with the 1964 Helsinki Declaration and its later amendments or comparable ethical standards.

\section{Consent for publication}

A written informed consent was obtained for all the patient.

\section{Copyright}

(c) The Author(s) 2021.

\section{REFERENCES}

1. Parkinson RJ, Eddleman CS, Batjer HH, Bendok BR. Giant intracranial aneurysms: endovascular challenges. Neurosurgery 2008;62:1336-45. DOI PubMed

2. Cagnazzo F, Mantilla D, Rouchaud A, et al. Endovascular treatment of very large and giant intracranial aneurysms: comparison between reconstructive and deconstructive techniques-A meta-analysis. AJNR Am J Neuroradiol 2018;39:852-8. DOI PubMed PMC

3. Piano M, Valvassori L, Quilici L, Pero G, Boccardi E. Midterm and long-term follow-up of cerebral aneurysms treated with flow diverter devices: a single-center experience. J Neurosurg 2013;118:408-16. DOI PubMed

4. Snyder KV, Natarajan SK, Hauck EF, et al. The balloon anchor technique: a novel technique for distal access through a giant aneurysm. J Neurointerv Surg 2010;2:363-7. DOI PubMed

5. Fargen KM, Velat GJ, Lawson MF, Hoh BL, Mocco J. The stent anchor technique for distal access through a large or giant aneurysm. $J$ Neurointerv Surg 2013;5:e24. DOI PubMed

6. Edwards L, Kota G, Morris PP. The sea anchor technique: a novel method to aid in stent-assisted embolization of giant cerebral aneurysms. J Neurointerv Surg 2013;5:e39. DOI PubMed

7. Parry PV, Morales A, Jankowitz BT. Solitaire salvage: a stent retriever-assisted catheter reduction technical report. BMJ Case Rep 2015;2015:bcr2014011336. DOI PubMed PMC

8. Quäschling U, Kläver M, Richter C, et al. Flow diversion in challenging vascular anatomies: the use of low profile stent retrievers for safe and accurate positioning of the microcatheter. CVIR Endovasc 2020;3:19. DOI PubMed PMC 